\title{
Grain boundary effects on plastic deformation and fracture mechanisms in Cu nanowires: Molecular dynamics simulations
}

\author{
Ajing Cao, ${ }^{1,2, *}$ Yueguang Wei, ${ }^{1}$ and En $\mathrm{Ma}^{2}$ \\ ${ }^{1}$ State Key Laboratory of Nonlinear Mechanics, Institute of Mechanics, Chinese Academy of Sciences, Beijing 100080, \\ People's Republic of China \\ ${ }^{2}$ Department of Materials Science and Engineering, Johns Hopkins University, Baltimore, Maryland 21218, USA
}

(Received 22 November 2007; revised manuscript received 21 March 2008; published 19 May 2008)

\begin{abstract}
Metallic nanowires have many attractive properties such as ultra-high yield strength and large tensile elongation. However, recent experiments show that metallic nanowires often contain grain boundaries, which are expected to significantly affect mechanical properties. By using molecular dynamics simulations, here, we demonstrate that polycrystalline $\mathrm{Cu}$ nanowires exhibit tensile deformation behavior distinctly different from their single-crystal counterparts. A significantly lowered yield strength was observed as a result of dislocation emission from grain boundaries rather than from free surfaces, despite of the very high surface to volume ratio. Necking starts from the grain boundary followed by fracture, resulting in reduced tensile ductility. The high stresses found in the grain boundary region clearly play a dominant role in controlling both inelastic deformation and fracture processes in nanoscale objects. These findings have implications for designing stronger and more ductile structures and devices on nanoscale.
\end{abstract}

DOI: 10.1103/PhysRevB.77.195429

PACS number(s): 61.46.Km, 61.72.Mm, 31.15.xv

\section{INTRODUCTION}

The use of metallic nanowires (NWs) as building blocks, templates, and connecting components in chemical and biological sensors, as well as in electronic and/or optical devices, demands a thorough understanding of their structures and mechanical properties. ${ }^{1,2}$ One important feature of metallic NWs is the surface effects due to their large surface to volume ratio. The surface-stress-induced intrinsic stress in the wire, in the order of gigapascals, leads to NW mechanical properties quite different from the bulk. ${ }^{3,4}$ In general, a benefit from reducing the sample size down to nanoscale is the decreased possibility of defects and flaws in material. However, recent experimental observations show that metallic NWs are often polycrystalline, ${ }^{5-9}$ typically containing grain boundaries (GBs), transecting the whole NW normal to its longitudinal axis (bamboo structure). For example, Wu et al. ${ }^{5}$ reported the transmission electron microscope (TEM) image of their electrochemically synthesized Au NWs, revealing that individual grains span, the NW diameter and are separated from adjacent grains by GBs. There are also intentionally designed heterogeneous structures in NWs, such as multilayered structures for magnetic properties, or sectioned wires made of different metals for functionalizing purposes. ${ }^{10,11}$ Due to difficulties associated with standard tensile or bending tests for single free-standing NW, the mechanical properties of these NWs containing boundaries and interfaces have not been evaluated. ${ }^{5}$

In this regard, atomistic simulation is a very useful tool to explore structure-property relationship on the nanoscale. ${ }^{12-15}$ Indeed, interesting phenomena regarding the yielding and fracture behavior of perfect single-crystalline metallic NWs have been uncovered by using atomistic simulations. ${ }^{16-22}$ However, studies on the structure and mechanical properties of polycrystalline NWs are still lacking. One intriguing issue of interest here is that when both of the two characteristic length scales (sample size and grain size) reach nanoscale, which size effect would be more important. In other words, in these polycrystalline NWs, both the free surface and GB interface are competing in controlling the plastic deformation. It is not immediately clear if deformation would be mediated by GB alone, or also by processes originated from free surfaces as in single-crystal NWs. The objective of this paper is to provide an atomistic understanding of the GB effects on the strength, ductility as well as fracture of the $\mathrm{Cu}$ NWs.

\section{SIMULATION MODELS}

Our molecular dynamics (MD) simulations employed bamboo-like polycrystalline $\mathrm{Cu}$ NWs with square crosssection, similar to the configuration in experiments. ${ }^{5} \mathrm{We}$ choose the [100] crystallographic direction as the misorientation axis. The GBs separating individual grains are $\Sigma 5(310) 36.9^{\circ}$ symmetric high-angle tilt GBs, which has a high density of coincident atomic sites across the interface plane. ${ }^{23}$ This type of GB is typical in the experiment observations ${ }^{24}$ and computer simulations. ${ }^{25}$ The distance between two adjacent GBs (mean grain size) is $8 \mathrm{~nm}$, which is comparable to the experimentally investigated grain size (5-40 nm). For comparison purposes, we also perform simulations of [100] single-crystalline $\mathrm{Cu} \mathrm{NW}$ with a square cross-section with $\{100\}$ side surfaces. These two wires have the same size, which is $8 \times 8 \times 32 \mathrm{~nm}^{3}$. An embedded-atom method potential for $\mathrm{Cu}$ by Mishin et al. ${ }^{26}$ is chosen in the present work because it is established based on $a b$ initio results of stacking fault and twin formation energies and used before for simulating GB properties. ${ }^{26}$ To discern defects in NWs, colors are assigned to the atoms according to a local crystallinity classification visualized by common neighbor analysis, ${ }^{27}$ which permits the distinction between atoms in a local hexagonal-close-packed (hcp) environment and those in face-centered-cubic (fcc) environment. ${ }^{15,21,22}$ 


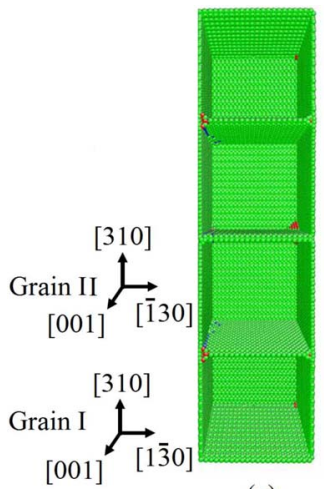

(a) (b)

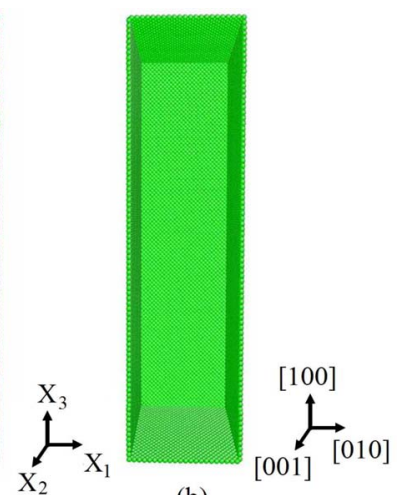

FIG. 1. (Color online) (a) Relaxed bamboolike polycrystalline $\mathrm{Cu} \mathrm{NW}$ and (b) [100] single-crystalline $\mathrm{Cu}$ NW. The perfect fcc atoms and the front surface are not shown. The crystallographic orientations of two bottom grains in polycrystalline $\mathrm{Cu} \mathrm{NW}$ are labeled.

After initial construction, energy minimization was performed by means of the conjugate gradient method. The wires were then thermally equilibrated to $300 \mathrm{~K}$ for $20 \mathrm{ps}$ using a Nosé-Hoover thermostat. ${ }^{28,29}$ No periodic boundary condition was utilized in all three dimensions. The relaxed polycrystalline NW and [100] single-crystalline NW is shown in Fig. 1. By starting from the equilibrium configuration of the NWs, uniaxial tensile loading was applied until failure. To speed up the simulation for efficiency, in the first seven loading steps, all atoms were displaced in a prescribed uniform strain increment of $0.5 \%$ in the length direction. Smaller $(0.2 \%)$ strain increment was applied in the remaining steps. For each step, the NW was relaxed for 120 ps with their ends fixed at the prescribed strain at a constant temperature of $300 \mathrm{~K}$. This equilibration process usually took less than $100 \mathrm{ps}$ and the average stress over the last $5 \mathrm{ps}$ of the relaxation period was taken as the stress of the NWs. The loading stain rate was on the order of $10^{7} \mathrm{~s}^{-1}$, which is rather slow for MD simulations. The stresses reported in this work are calculated based on the Virial theorem, which is equivalent to the Cauchy stress in the average sense. ${ }^{30}$

\section{RESULTS AND DISCUSSION}

The stress-strain $(\sigma-\varepsilon)$ curves obtained for the polycrystalline NW and the [100] single-crystalline NW are shown in Fig. 2. Both wires show linear stress-strain response during the initial elastic deformation. The calculated Young's moduli $(E)$ for the two curves are 70 and $80 \mathrm{GPa}$, respectively. In the case of single-crystalline NW, after the elastic deformation, the stress shows precipitous drop indicating yielding. The most striking feature of the mechanical response of the single-crystalline NW is the serrated nature of the stress-strain curve. This can be attributed to sequential alternating events of dislocation nucleation, propagation, and escape from the wire, with dislocation starvation in the interior of the wires. Actually, in the absence of GBs, our simulation shows that all dislocations are nucleated from the surface edges in the single crystalline wires. Once dislocation

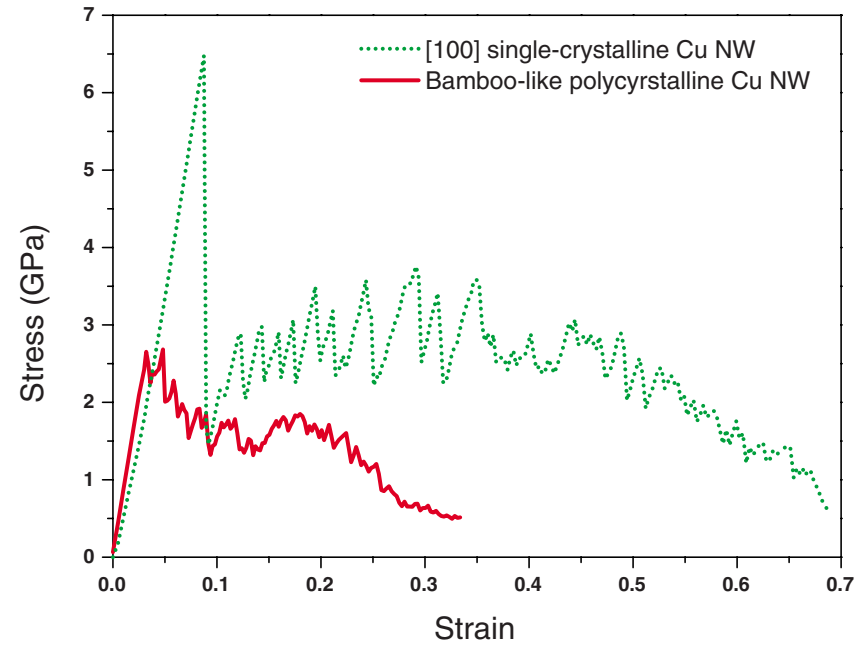

FIG. 2. (Color online) Stress-strain curves of the bamboo-like polycrystalline $\mathrm{Cu} \mathrm{NW}$ and the [100] single-crystalline $\mathrm{Cu}$ NW.

nucleates from surface edges, it can only travel much shorter distances before annihilated at the free surface, thereby reducing the probability of multiplication processes. Eventually, this leads to a state of dislocation starvation in the wire interior. Then, elastic deformation is required to nucleate new dislocations, corresponding to the following yielding. These processes repeat and result in a serrated stress-strain response (see Fig. 1). Also, after the first yielding, there is always left dislocation slip lines on the free surfaces, which are the most favorable sites for dislocation nucleation, and correspondingly, the required stress for the following yielding is much lower than for the initial one.

In contrast, the mechanical response of polycrystalline NW shows several significantly different features. First, the initial yielding only leads to a small stress reduction. Second, the wavy features in the stress-strain curve are far less distinct compared with that in the [100] single-crystalline NW. In Fig. 2, both the yield stress and the fracture strain of the polycrystalline NWs are significantly lower than those of the single-crystalline wire. The yield stress of the polycrystalline wire is $2.6 \mathrm{GPa}$, while the yield stress of the singlecrystalline wire is $6.4 \mathrm{GPa}$ (approaching the theoretical value of E/10). The ultimate fracture strain of the polycrystalline wire is 0.34 but for the single-crystalline wire the fracture strain is 0.68 .

However, while the misorientation axis is in the [100] direction after forming the bamboo structure, the polycrystalline wire axis is now along the [310] direction (required to created the symmetric tilt grain boundary). To observe the effect of crystallographic orientation on the yield stress, ${ }^{31}$ we have also performed simulations on the [310] singlecrystalline wires with the same size. The tensile yield stress is $4.0 \mathrm{GPa}$, which is also much higher than that of the bamboo wire. After yielding, the stress quickly drop to $2.3 \mathrm{GPa}$, which is much similar to the [100] single-crystalline one. This rules out the possibility that the reduced strength of the polycrystalline wires is mainly due to the different wire orientation. It is the different dislocation nucleation mechanisms that are responsible for the strength differences, as discussed below. 
In a conventional polycrystalline metal, the bulk stresses arising from interface stresses are negligible. However, for a metallic NW with nanometer-scale grains, GB interface stresses are important because they induce bulk stresses on the order of $f / L$, where $f$ is the scalar interface stress and $L$ denotes a characteristic length scale of the microstructure (such as the average grain size here) ${ }^{32}$ To elucidate the origin of the role of GBs in reducing the yield stress, the distribution of atomic-level stress component is examined. This local stress in the relaxed wire is meaningful in the case of $T=0 \mathrm{~K}$ since the thermal fluctuation is eliminated. The atomic-level stress associated with atom $i$ is obtained by decomposing the Virial stress onto individual atoms,

$$
\Pi^{\alpha \beta}=\frac{1}{\Omega} \sum_{i} \omega_{i} \pi_{i}^{\alpha \beta},
$$

where

$$
\pi_{i}^{\alpha \beta}=\frac{1}{\omega_{i}}\left[-m_{i} v_{i}^{\alpha} v_{i}^{\beta}+\frac{1}{2} \sum_{j \neq i}\left(-\frac{1}{r_{i j}} \frac{\partial U_{\text {total }}}{\partial r_{i j}}\right) r_{i j}^{\alpha} r_{i j}^{\beta}\right]
$$

and $\Sigma \omega_{i}=\Omega$, where $\omega_{i}$ is taken as the effective volume of the atom $i, \Omega$ is the volume of the system, $m_{i}$ and $v_{i}$ are the mass and velocity of atom $i$, the indices $\alpha$ and $\beta$ denote the Cartesian components. $r_{i j}^{\alpha}$ is the projection of the interatomic distance vector $\mathbf{r}_{i j}=\mathbf{r}_{i}-\mathbf{r}_{j}$ along coordinate $\alpha$, and $r_{i j}=\left|\mathbf{r}_{i j}\right| .^{20,21}$ The Virial stress for an atom is defined ${ }^{20,21}$ as

$$
\Pi^{\alpha \beta}=\frac{1}{\Omega}\left\{-\sum_{i} m_{i} v_{i}^{\alpha} v_{i}^{\beta}+\frac{1}{2} \sum_{i} \sum_{j \neq i}\left(-\frac{1}{r_{i j}} \frac{\partial U_{\text {total }}}{\partial r_{i j}}\right) r_{i j}^{\alpha} r_{i j}^{\beta}\right\} .
$$

Due to the high surface to volume ratio, the free standing NWs are not in equilibrium and will undergo contraction in the length direction, this relaxation mainly occurred in the energy minimization process at $T=0 \mathrm{~K}$ in our case, and also causes changes in the GB structure and introduces stresses. For illustration, the distribution of normal stress component $\left(\pi^{33}\right)$ along the relaxed wire axis is shown in Fig. 3(a). The stresses near GB interfaces are quite different from those of the grain interior. Typically, unlike the surface stresses, the interface stress is a tensor with components that have opposing sign across a GB interface. Figure 3(b) shows that the stresses are quite uniform in the wire interior far from the GBs except near the free surfaces. Figure 3(c) shows the distribution of the heterogeneous interface stresses in the GB region, which directly result from the large fractions of disordered atoms at the GB. Meanwhile, the magnitudes of stresses at the GB interface are much larger than the free surface induced stresses in the wire interior, the maximum tensile and compressive values being as high as $25.0 \mathrm{GPa}$ and $-20.0 \mathrm{GPa}$, respectively. By averaging the atomic stress component $\pi^{33}$ over the atoms in the GB region, the averaged stress is $1.4 \mathrm{GPa}$ (tensile). The maximum normal component of tensile surface stress is $\sim 5.8 \mathrm{GPa}$ (see Fig. 3). The stress in the wire interior in the middle of the grains, which is induced by both the surface stresses and interface stresses, is about $-0.8 \mathrm{GPa}$ (compressive).

A close inspection of the NW deformation snapshots shows that the onset of plasticity in polycrystalline NWs is via nucleation of partial dislocations from the GB interface

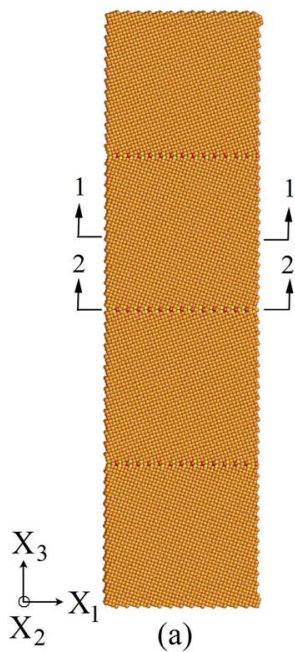

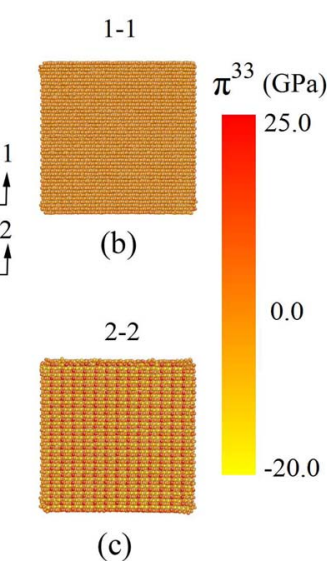

(c)

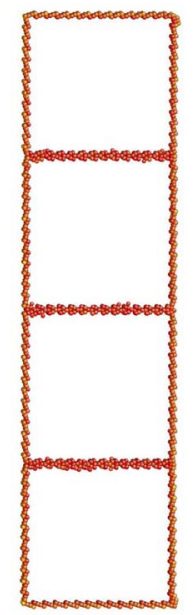

(d)
FIG. 3. (Color online) Comparison of interface stresses and stresses in the grain interior of the polycrystalline $\mathrm{Cu} N W$ at $T=0 \mathrm{~K}$. (a) Distribution of normal stress component $\left(\pi^{33}\right)$ along the relaxed wire axis. (b) Distribution of normal stress component $\left(\pi^{33}\right)$ on the cross-section 1-1, showing uniform stress distribution in the middle of the grain except free surfaces. (c) Distribution of normal stress component $\left(\pi^{33}\right)$ on the cross-section 2-2, showing heterogeneous interface stress distribution in the GB region. (d) Atoms with potential energy higher than $-2.5 \mathrm{eV}$.

on two different slip systems in the two neighboring grains. In Fig. 4(a), the first leading partial dislocations are nucleated from GBs, with stacking faults left behind, as indicated by black arrows. In Figs. 4(b)-4(d), the partial dislocations move on the primary $\{111\}$ slip system across the whole wire and are then absorbed by the free surfaces and the opposing GBs. This deformation process leads to a dislocation-free grain interior. Further straining requires the nucleation of other dislocations from GBs.

The high heterogeneous interface stresses (of the order of gigapascals) due to the presence of disordered atoms at GB are consistent with the high energies of the GB atoms, as shown in Fig. 3(d). They are directly responsible for the nucleation of dislocations from the GB sources. This is the origin of the much lowered yield stress in the polycrystalline NWs. In contrast, the yielding mechanism of the perfect defect-free single-crystalline NWs is via the collective nucleation (and subsequent propagation) of partial dislocations from free surface edges, which requires stresses closer to theoretical strength. These two dramatically different dislocation nucleation mechanisms are responsible for the different yields and flow behavior of the two types of NWs. In other words, competition between dislocation nucleation from GBs and from free surfaces is the governing physics controlling the plastic deformation mechanisms of the NWs with GBs. For metallic NWs with nanoscale grains, the role of GB character on material properties is amplified due to the increased fraction of atoms that are positioned at the GB interfaces.

The critical shear stress for dislocation nucleation is quite different for the wires with and without GBs, which indicates that the yielding criterion for metallic NWs is more closely associated with local resolved shear stress (RSS) distribution 


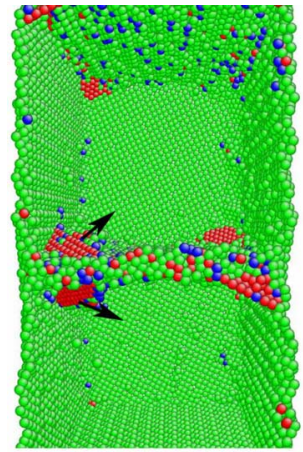

(a)

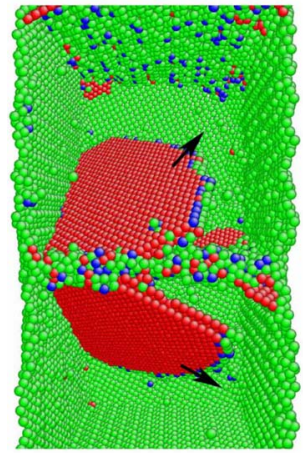

(c)

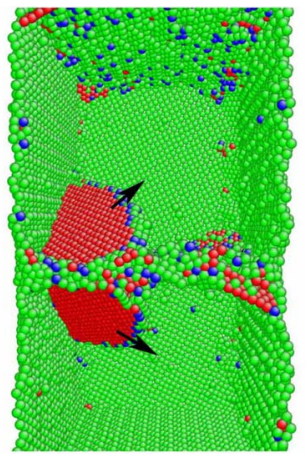

(b)

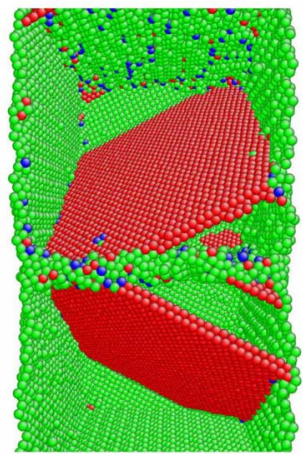

(d)
FIG. 4. (Color online) A series of sequential snapshots showing the onset of yielding in $\mathrm{Cu}$ polycrystalline NWs via partial dislocation emission from GBs. (a) First leading partials nucleating from the symmetric GB interface in the adjacent grains. [(b)-(c)] Dislocations propagating in the grains. (d) Partial dislocations reaching free surface and their incorporation in GBs with stacking faults left behind. Perfect fcc atoms and the front surface are not shown for visualizing the inner defects. The black arrows indicate partial dislocations.

rather than the average RSS in the wire interior, as proposed by Diao et al. ${ }^{20}$ The structure of the GBs region is therefore very critical. Essentially, the loss of some nearest neighbors causes the atoms in the GB environment to behave in a heterogeneous manner, leading to high intrinsic interface stresses in the GBs and hence a much lower apparent yield stress of the polycrystalline NWs.

Figure 5(a) shows the detailed deformation process of polycrystalline NW during tensile loading, which clearly shows necking at GBs. At the strain of 0.08 , the necking instability ${ }^{33}$ set in at the GB, causing limited uniform deformation and eventually failure. In contrast, the failure of the [100] single-crystalline NW is via the stable growth of shear offset. There, a large number of dislocations nucleate from free surface edges and two major slip planes are favorably activated during stretching. As a result, the dislocations gliding on the slip planes lead to slip bands on the free surfaces. These slip bands are then the preferred dislocation nucleation sites in subsequent deformation, which leads to shear failure in a gradual manner. The typical deformation process for the [100] single-crystalline Cu NW is shown in Fig. 5(b).

It is not surprising to observe necking initiation at GBs, as the GB with distorted structure and excess volume is the weak link and hence the preferred site for stain localization. (a)
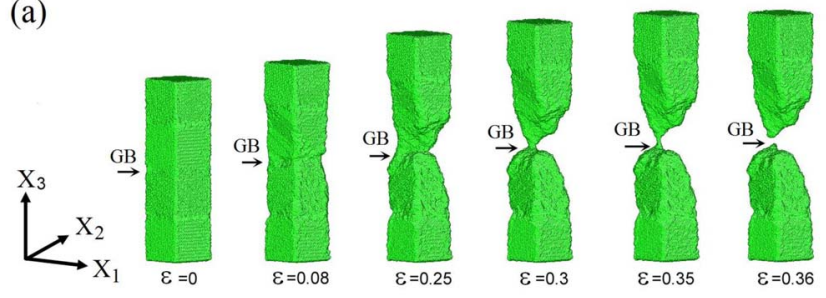

(b)
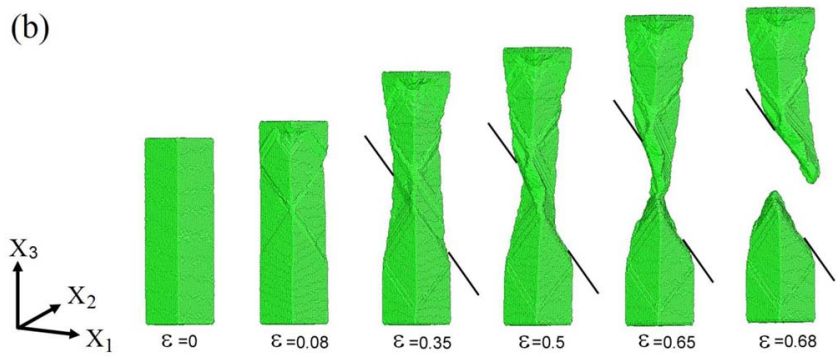

FIG. 5. (Color online) Different failure processes of polycrystalline and single crystalline $\mathrm{Cu}$ NWs. (a) Snapshots of the deformation of the polycrystalline $\mathrm{Cu}$ NW showing necking at GB. GB interface at the middle of the wire is indicated with black arrow. (b) Snapshots of the deformation of the [100] single-crystalline $\mathrm{Cu}$ $\mathrm{NW}$, showing stable growth of shear offset before ultimate failure. The black lines indicate the shear plane.

This instability leads to a much lower tensile ductility ${ }^{33} \mathrm{com}-$ pared to that of a perfect single-crystalline wire. It was shown recently that $\mathrm{Cu}$ NWs break up along the transverse GBs during thermal annealing at $400{ }^{\circ} \mathrm{C} .{ }^{9}$ These experimental results are consistent with our finding that GBs are the favorable failure sites.

We note that coherent twin boundaries (TBs) have been found to strengthen, in both experiments ${ }^{34}$ and atomistic simulations. ${ }^{21,22,35,36}$ However, these perfectly coherent TBs are special GBs that do not emit dislocations but block dislocation motion. The twin structure also changes the intrinsic stress distribution due to the change of grain orientation so that larger external stress is required to make these twinned wires yield. This is a scenario quite different from the incoherent $\Sigma 5(310) 36.9^{\circ}$ symmetric high angle tilt GBs simulated in this work, which is more typical of general highangle GBs. The high-energy GBs are also where the impurities tend to segregate to and where higher atomic mobility is expected to facilitate various activated processes. ${ }^{37,38}$

Nanoscale grain size ( $8 \mathrm{~nm}$ here) of the polycrystalline $\mathrm{Cu}$ NWs precludes the possibility of interaction and pile up of dislocations, as they are easily incorporated by near GBs or free surfaces a short distance away. The mechanism of dislocation storage for strain hardening is thus not seen in this polycrystalline NWs.

\section{CONCLUSION}

In summary, we have performed atomistic simulations to investigate how GBs affect plastic deformation mechanism and fracture processes of bamboolike $\mathrm{Cu}$ NWs. The highest intrinsic stresses and atoms of high energy are located at the 
GB interface due to its heterogeneous structure. This leads to mechanical properties quite different from those of singlecrystal NWs. The yielding mechanism is via the nucleation and propagation of partial dislocation from GB interfaces rather than from free surfaces, causing the yield strength to be much lower than that of perfect single-crystalline wires. GBs are also the preferential sites to initiate necking, which are accompanied by local atomic shuffling after dislocation emission from GBs, such that the fast reduction of crosssectional area at GBs significantly decreases the tensile ductility of the wire, resulting in limited uniform deformation and accelerating fracture. Our MD results indicate that between the two types of defects, GBs and free surfaces, GBs play more dominant role in controlling the mechanical be- havior of NWs, at least for the particular wire size and GB features simulated here. Therefore, microstructure control remains critical in nanoscaled objects to ensure the desired mechanical properties.

\section{ACKNOWLEDGMENTS}

A.C. and Y.W. are supported by National Science Foundation of China through Grants No. 10432050, No. 10721202, and No. 10672163. We acknowledge S. Plimpton of Sandia National Laboratory for sharing his MD code PARADYN. ${ }^{39}$ E.M. is supported at JHU by NSF Grant No. DMR-0355395.
*Corresponding author; ajcao@jhu.edu

${ }^{1}$ E. W. Wong, P. E. Sheehan, and C. M. Lieber, Science 277, 1971 (1997).

${ }^{2}$ A. P. Suryavanshi and M. F. Yu, Appl. Phys. Lett. 88, 083103 (2006).

${ }^{3}$ J. Diao, K. Gall, and M. L. Dunn, Nat. Mater. 2, 656 (2003).

${ }^{4}$ J. Diao, K. Gall, and M. L. Dunn, Nano Lett. 4, 1863 (2004).

${ }^{5}$ B. Wu, A. Heidelberg, and J. J. Boland, Nat. Mater. 4, 525 (2005).

${ }^{6}$ A. Bietsch and B. Michel, Appl. Phys. Lett. 80, 3346 (2002).

${ }^{7}$ W. Wu, S. H. Brongersma, M. V. Hove, and K. Maex, Appl. Phys. Lett. 84, 2838 (2004).

${ }^{8}$ L. Klinger and E. Rabkin, Acta Mater. 54, 305 (2006).

${ }^{9}$ M. E. Toimil Molares, A. G. Balogh, T. W. Cornelius, R. Neumann, and C. Trautmann, Appl. Phys. Lett. 85, 5337 (2004).

${ }^{10}$ A. K. Salem, J. Chao, K. W. Leong, and P. C. Searson, Adv. Mater. (Weinheim, Ger.) 16, 268 (2004).

${ }^{11}$ B. Wildt, P. Mali, and P. C. Searson, Langmuir 22, 10528 (2006).

${ }^{12}$ V. Yamakov, D. Wolf, S. R. Phillpot, A. K. Mukherjee, and H. Gleiter, Nat. Mater. 1, 45 (2002).

${ }^{13}$ H. V. Swygenhoven, Science 296, 66 (2002).

${ }^{14}$ J. Schiøtz and K. W. Jacobsen, Science 301, 1357 (2003).

${ }^{15}$ A. J. Cao and Y. G. Wei, Appl. Phys. Lett. 89, 041919 (2006).

${ }^{16}$ W. Liang, M. Zhou, and F. Ke, Nano Lett. 5, 2039 (2005).

${ }^{17}$ W. Liang and M. Zhou, Phys. Rev. B 73, 115409 (2006).

${ }^{18}$ H. S. Park, K. Gall, and J. A. Zimmerman, Phys. Rev. Lett. 95, 255504 (2005).

${ }^{19}$ K. Gall, J. Diao, and M. L. Dunn, Nano Lett. 4, 2431 (2004).

${ }^{20}$ J. Diao, K. Gall, M. L. Dunn, and J. A. Zimmerman, Acta Mater.
54, 643 (2006).

${ }^{21}$ A. Cao and Y. G. Wei, Phys. Rev. B 74, 214108 (2006).

${ }^{22}$ A. J. Cao, Y. G. Wei, and S. X. Mao, Appl. Phys. Lett. 90, 151909 (2007).

${ }^{23}$ J. M. Howe, Interfaces in Materials (Wiley, New York, 1997).

${ }^{24}$ K. L. Merkle, Interface Sci. 2, 311 (1995).

${ }^{25}$ D. E. Spearot, M. A. Tschopp, K. I. Jacob, and D. L. McDowell, Acta Mater. 55, 705 (2007).

${ }^{26}$ Y. Mishin, M. J. Mehl, D. A. Papaconstantopoulos, A. F. Voter, and J. D. Kress, Phys. Rev. B 63, 224106 (2001).

${ }^{27}$ J. D. Honeycutt and H. C. Andersen, J. Phys. Chem. 91, 4950 (1987).

${ }^{28}$ S. Nosé, Mol. Phys. 52, 255 (1984).

${ }^{29}$ W. G. Hoover, Phys. Rev. A 31, 1695 (1985).

${ }^{30}$ K. S. Cheung and S. Yip, J. Appl. Phys. 70, 5688 (1991).

${ }^{31}$ M. A. Tschopp, D. E. Spearot, and D. L. McDowell, Modell. Simul. Mater. Sci. Eng. 15, 693 (2007).

${ }^{32}$ R. Birringer, M. Hoffmann, and P. Zimmer, Phys. Rev. Lett. 88, 206104 (2002).

${ }^{33}$ E. Ma, Nat. Mater. 2, 7 (2003).

${ }^{34}$ B. Wu, A. Heidelberg, J. J. Boland, J. E. Sader, X. M. Sun, and Y. D. Li, Nano Lett. 6, 468 (2006).

${ }^{35}$ A. J. Cao and Y. G. Wei, J. Appl. Phys. 102, 083511 (2007).

${ }^{36}$ Z. H. Jin, P. Gumbsch, E. Ma, K. Albe, K. Lu, H. Hahn, and H. Gleiter, Scr. Mater. 54, 1163 (2006).

${ }^{37}$ E. Ma, M.-A. Nicolet, and M. Natan, J. Appl. Phys. 65, 2703 (1989).

${ }^{38}$ E. Ma, C. V. Thompson, and L. A. Clevenger, J. Appl. Phys. 69, 2211 (1991).

${ }^{39}$ S. J. Plimpton, J. Comput. Phys. 117, 1 (1995). 